\title{
Studies of TMDs at COMPASS
}

\author{
H. Wollny on behalf of the COMPASS Collaboration \\ Physikalisches Institut, Albert-Ludwigs University of Freiburg, \\ 79104 Freiburg, Germany \\ *Heiner.Wollny@cern.ch
}

\begin{abstract}
Transverse spin and transverse momentum distribution functions of the constituents of the nucleon are a crucial input for a complete description of the nucleon. COMPASS measured such for longitudinally and transversely polarized deuterons and protons. In the following we will focus on recent results from the 2007 transverse proton data and on the results for unpolarized deuterons.

Keywords: SIDIS, polarized target, Transversity, TMD, Collins, Sivers
\end{abstract}

\section{Introduction}

At leading order and integrating over transverse quark momenta, three parton distribution functions (PDFs) are needed for a complete description of the nucleon. The quark density $q(x)$, the helicity distribution $\Delta q(x)$ and the transversity distribution $\Delta_{T} q(x)$. The first PDF, $q(x)$, describes the probability to scatter off a quark inside the nucleon carrying the momentum fraction $x$. The helicity PDF, $\Delta q(x)$, is defined for a longitudinal polarized proton as the difference of the probabilities that the struck quark carries momentum fraction $x$ and its spin is parallel or anti-parallel to the spin of the parent nucleon. The transversity PDF, $\Delta_{T} q(x)$, is analogously defined to $\Delta q(x)$, however for a transversely polarized nucleon. Because $\Delta_{T} q(x)$ is chiral-odd it cannot be measured in inclusive DIS. However it can be accessed in semi-inclusive deep-inelastic scattering in combination with a chiral-odd fragmentation function. Three different channels to address the transversity distribution, have been analysed, both for deuteron and proton targets. They will be discussed in Sec. 3 .

If one considers intrinsic transverse momenta of the quarks inside the nucleon, several further PDFs, so called transverse momentum dependent distribution functions (TMDs), contribute to the SIDIS cross-section, which will be discussed in Sec. 4 . 


\section{The COMPASS Experiment}

COMPASS is a fixed target experiment situated at the M2 beam line of the SPS accelerator at CERN. It has a wide physics program dedicated to the study of the nucleon spin structure and of hadron spectroscopy. In the years 2002, 2003, 2004 and 2006 data with a polarized ${ }^{6} \mathrm{LiD}$ target were taken. In the years 2002-2004 approximately $20 \%$ of the data taking was dedicated to transverse target polarization. In the year 2007 COMPASS took data with a $\mathrm{NH}_{3}$ target, equally shared between longitudinal and transverse target polarization. After the years 2008 and 2009, dedicated to baryon and meson spectroscopy measurements with hadron beams, in 2010 COMPASS continues with muon beam and transversely polarized protons.

The target consists of several cells along the beam direction, which are oppositely polarized. The polarization is periodically reversed to reduce systematic effects due to the different acceptances of the cells. The detector consists of two open field dipole magnets, allowing the detection of particles with \pm 180 mrad. Particle identification is done with a Ring Imaging Cerenkov detector, two hadron calorimeters and muon filters. For a detailed description we refer to reference [1].

\section{Transversity}

Collins Asymmetry: The chiral-odd transversity distribution can be measured in SIDIS involving the chiral-odd Collins fragmentation function $\Delta_{T}^{0} D_{q}^{h}[2]$, which describes the fragmentation of transversely polarized quarks into unpolarized hadrons. According to Collins the fragmentation leads to an asymmetry $A_{\text {Coll }}$ of the number of produced hadrons in $\sin \left(\phi_{h}+\phi_{S}-\pi\right)$. Here $\phi_{h}$ is the azimuthal angle, measured around the direction of the virtual photon, of the hadron with respect to the scattering plane and $\phi_{S}$ is the azimuthal angle between the spin of the initial quark and the scattering plane $[3-5]$. In first order the measured asymmetry $A_{C o l l}$ is proportional to a convolution over intrinsic quark transverse momenta of the transversity distribution and the Collins fragmentation function measured at Belle [6]. Because of the convolution, assumptions about the transverse momentum dependence of the distribution and the fragmentation functions have to be made to extract transversity [7]. In addition the $Q^{2}$ evolution of the Collins fragmentation function from Belle to COMPASS energies is not known, introducing a further uncertainty.

Dihadron Interference: Another possibility to access the transversity distribution is to measure it in SIDIS in combination with the polarized 
dihadron interference fragmentation function (DiFF) [8-11]. Here, the fragmentation of a transversely polarized quark into two unpolarized hadrons leads to an azimuthal modulation in $\sin \left(\phi_{R}+\phi_{S}-\pi\right)$ in the number of produced hadron pairs. Here $\phi_{S}$ is defined as above and $\phi_{R}$ is the azimuthal angle, measured around the direction of the virtual photon, between the vector $\vec{R}=\left(z_{2} \vec{P}_{1}-z_{1} \vec{P}_{2}\right) /\left(z_{1}+z_{2}\right)$ and the scattering plane. Where $P_{1}$ and $P_{2}$ are the momenta of the two hadrons and $z_{1}$ and $z_{2}$ are their fractional energies. For oppositely charged hadron pairs $P_{1}$ is per definition the momentum of the hadron with positive charge, otherwise $P_{1}$ is the momentum of the hadron with the largest energy fraction. Here, the measured asymmetry $A_{R S}$ is proportional to a product of the transversity distribution and the polarized DiFF, recently measured at Belle [12]. Therefore, no assumptions about transverse momentum dependences of distribution and fragmentation functions are needed, to extract transversity. In addition, the $Q^{2}$ evolution of the polarized DiFF from Belle energies to COMPASS energies is known [13]. Hence, the extraction of transversity with this channel is from theoretical point of view cleaner than the extraction from the Collins asymmetries.

Lambda Polarization: A third channel to measure transversity is to study $\Lambda$ and $\bar{\Lambda}$ polarization in SIDIS with a transversely polarized target [14]. The polarization $P_{T}^{\Lambda}$ of the produced $\Lambda / \bar{\Lambda}$-hyperons, measured via the parity violating decay into $\Lambda \rightarrow p \pi^{-}$and $\bar{\Lambda} \rightarrow \bar{p} \pi^{+}$, respectively, is used as a polarimeter of the initial transverse spin of the fragmenting quark. It is in first order proportional to a product of transversity and the fragmentation function $\Delta_{T} D_{q}^{\Lambda}$, which describes the fragmentation of a transversely polarized quark into a transversely polarized $\Lambda$-hyperon.

\section{Transverse Momentum Dependent Distribution Functions, TMDs}

Taking into account intrinsic transverse momenta of the quarks in total eight TMDs are needed for a complete description at leading-twist [15]. For unpolarized nucleons the Cahn effect, the Boer-Mulders TMD and perturbative QCD effects, like gluon radiation, contribute to $\cos \phi_{h}$ and $\cos 2 \phi_{h}$ modulations in the SIDIS cross section of single hadrons [16-18]. For a transversely polarized nucleon the Sivers TMD $\Delta_{0}^{T} q[19]$ is related to the quark angular orbital momentum inside a transversely polarized nucleon and is therefore of special interest since this could be a crucial piece to solve the nucleon spin puzzle. The Sivers effect leads to an azimuthal modulation of the number of produced hadrons in $\sin \left(\phi_{h}-\phi_{S}\right)$, where $\phi_{h}$ and 
$\phi_{S}$ are defined as for the Collins asymmetry discussed in Sec. 3. The asymmetry $A_{S i v}$ is proportional to a convolution over intrinsic quark transverse momenta of the Sivers function and the well-known unpolarized fragmentation function $D_{q}^{h}$.

Recent results of the Sivers function measured with protons and results of $\cos \phi_{h}$ and $\cos 2 \phi_{h}$ modulations for unpolarized deuterons will be reviewed in this article. For the six remaining azimuthal asymmetries measured with transversely polarized deuterons the reader is referred to [20]. Detailed results of azimuthal asymmetries measured with longitudinally polarized deuterons can be found in [21].

\section{Event selection}

Kinematic cuts on the squared four momentum transfer $Q^{2}>1(\mathrm{GeV} / c)^{2}$, on the fractional energy transfer of the muon $0.1<y<0.9$ and the hadronic invariant mass $W>5 \mathrm{GeV} / c^{2}$ are applied to select DIS events. The selection of charged hadrons differs slightly for the various analyses. However, for the single hadron analyses in general a fractional energy $z>0.2$ of the observed final hadron is required to select hadrons in the current fragmentation region. In addition a transverse momentum $p_{T}^{h}>0.1 \mathrm{GeV} / c$ of the hadron with respect to the virtual photon is required to ensure a good definition of $\phi_{h}$. Muons are rejected in demanding a minimal energy deposit in the hadronic calorimeters.

For the hadron pair analysis $z_{1,2}>0.1$ is required for each hadron and for the sum $z_{1}+z_{2}<0.9$ to reject exclusively produced $\rho^{0}$-mesons. In order to have a good definition of $\phi_{R}$ a cut on $R_{T}>70 \mathrm{MeV} / c$, the transverse component of $\vec{R}$ with respect to the virtual photon direction, is applied.

\section{Results}

\subsection{Transversity}

The Collins asymmetries $A_{\text {Coll }}^{p}$ have been evaluated for charged hadrons in bins of Bjorken $x$, energy fraction $z$ and transverse momentum $p_{T}^{h}$. The results for the full 2007 proton statistics [22] are shown in Fig. 1. For positive hadrons the asymmetry is negative and for negative hadrons it is positive. For both charges the size of the asymmetry increases with $x$ and is compatible in their strengths. The values agree both in magnitude and in sign with the previous measurements of HERMES [23], which have been performed at a considerably lower electron beam momentum of $27.5 \mathrm{GeV} / c$. From the transversely polarized deuteron data Collins asym- 
metries for unidentified charged hadrons, charged pions and charged kaons have been extracted [3-5]. All asymmetries found to be small and compatible with zero within the statistical errors. Hence for deuteron the transversity distribution must be small or even vanishing due to isospin symmetry, because the proton results and the Belle results [6] confirmed the existence of a non-zero Collins fragmentation function. The results for the transverse 2007 proton data of the dihadron asymmetries $A_{R S}^{p}$ for oppositely charged hadron pairs [24] are shown as a function of $x, z$ and $M_{i n v}$ in Fig. 2. A strong asymmetry is observed in the valence $x$-region. In the invariant mass the asymmetry is negative over the whole range. Compared to the Collins asymmetries, the size of $A_{R S}^{p}$ is approximately a factor of two larger, emphasizing the good analyzing power of the dihadron interference fragmentation function to measure transversity. From the transversely polarized

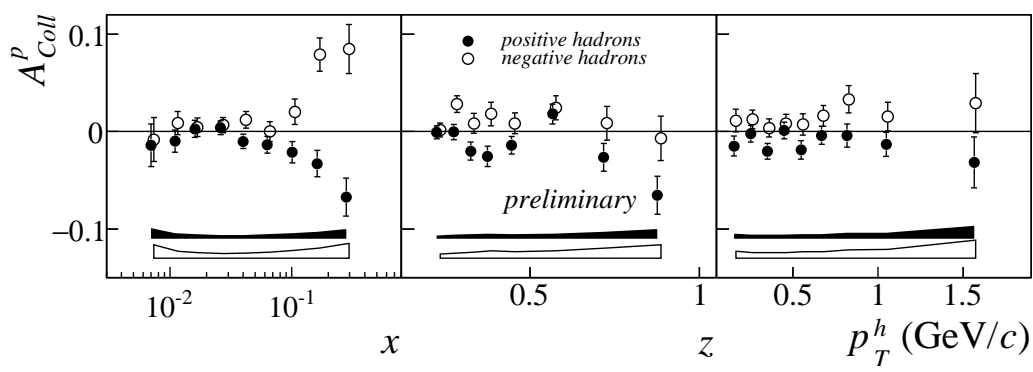

Fig. 1. Collins asymmetries for positive and negative hadrons as a function of $x, z$ and $p_{T}$. The horizontal bands indicate the systematic uncertainties.



Fig. 2. Dihadron asymmetries $A_{R S}^{p}$ for oppositely charged hadron pairs as a function of $x, z$ and $M_{i n v}$. The horizontal band indicates the systematic uncertainties.

deuteron data COMPASS extracted dihadron asymmetries for unidentified $h^{+} h^{-}$-pairs and identified $\pi^{+} \pi^{-}, K^{+} K^{-}, \pi^{+} K^{-}$and $K^{+} \pi^{-}$-pairs [25-27]. In addition also different charge combinations have been analysed, consid- 
ering the two most energetic hadrons in each event. All asymmetries found to be small and compatible with zero within the statistical errors.

The polarization of $\Lambda$ and $\bar{\Lambda}$-hyperons measured with transversely polarized ${ }^{6} \mathrm{LiD}$ and $\mathrm{NH}_{3}$ targets, respectively, have been evaluated in bins of $x$ and $z[28,29]$. For both targets the evaluated polarizations of $\Lambda$ and $\bar{\Lambda}$-hyperons are small and compatible with zero within the errors. This indicates, taking into account the sizeable Collins and dihadron asymmetries measured for protons, that the analyzing power of this channel seems to be small.

\subsection{Sivers-Function}

The Sivers asymmetries for charged hadrons from the transverse 2007 proton data [22] are shown, as a function of $x, z$ and $p_{T}^{h}$, in Fig. 3. In addition to the indicated systematic uncertainty, for positive hadrons an additional systematic uncertainty of \pm 0.01 is assigned reflecting a time variation of the spectrometer acceptance between the first and the second half of data taking used for this analysis. The asymmetry for positive hadrons is significantly positive over almost the complete $x$-range. The asymmetry for negative hadrons is small and compatible with zero within the statistical errors. Both results show the same trend as the previous measurement of HERMES [30]. However, the magnitude of the asymmetry for positive hadrons is smaller than the one of HERMES. This might be explained by a dependence on $W$, the invariant mass of the photon-nucleon system. Studies indicate that the asymmetry seems to be only sizeable at small $W$, where HERMES measures and approach zero at high $W$. The Sivers asymmetries for unidentified charged hadrons, charged pions and kaons extracted from transversely polarized ${ }^{6} \mathrm{LiD}[3-5]$ were all found to be small and compatible with zero within the statistical errors.

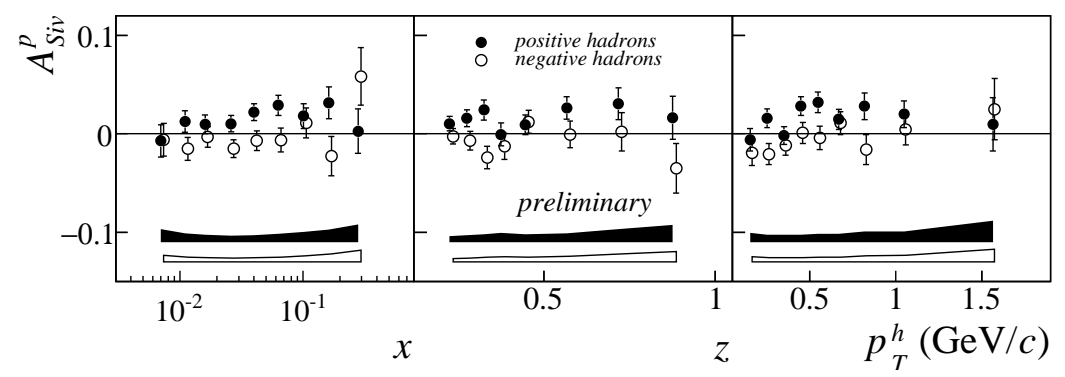

Fig. 3. Sivers asymmetries for positive and negative hadrons as a function of $x, z$ and $p_{T}$. The horizontal bands indicate the systematic uncertainties. 


\subsection{Azimuthal Asymmetries from Unpolarized Deuterons}

An unpolarized deuteron sample is obtained by combining data samples with opposite target polarization taken in the year 2004. The magnitudes of $\cos \phi_{h}$ and $\cos 2 \phi_{h}$ modulations for charged hadrons [31] as a function of $x, z$ and $p_{T}$ are shown in Fig. 4. Large asymmetries up to $20 \%$ are found for the $\cos \phi_{h}$ modulation and asymmetries in the order of $5 \%$ for the $\cos 2 \phi_{h}$ modulation. The trend of the asymmetries for positive and negative hadrons are similar, however, the magnitudes of the two differs significantly. The systematic uncertainties have been evaluated to be sizeable. The dominant contribution is given by the acceptance corrections determined with Monte Carlo simulations.



Fig. 4. Unpolarized asymmetries for charged hadrons as a function of $x, z$ and $p_{T}$. The kinematical factors are: $\epsilon_{1}=(2-y) \sqrt{1-y} /\left(1+(1-y)^{2}\right)$ and $\epsilon_{2}=(1-y) /\left(1+(1-y)^{2}\right)$.

\section{Summary and Outlook}

Recent results of COMPASS measurements related to transversity and transverse momentum dependent distribution functions have been presented. In the year 2010 and 2011 COMPASS will continue its measurements with transversely and longitudinally polarized protons, which will significantly reduce the statistical errors. 


\section{References}

1. P. Abbon et al., Nucl. Instrum. Meth. A577, 455 (2007).

2. J. C. Collins, Nucl. Phys. B396, 161 (1993).

3. V. Y. Alexakhin et al., Phys. Rev. Lett. 94, p. 202002 (2005).

4. E. S. Ageev et al., Nucl. Phys. B765, 31 (2007).

5. M. Alekseev et al., Phys. Lett. B673, 127 (2009).

6. R. Seidl et al., Phys. Rev. D78, p. 032011 (2008).

7. M. Anselmino et al., Nucl. Phys. Proc. Suppl. 191, 98 (2009).

8. A. V. Efremov et al., Phys. Lett. B284, 394 (1992).

9. X. Artru and J. C. Collins, Z. Phys. C69, 277 (1996).

10. M. Radici, R. Jakob and A. Bianconi, Phys. Rev. D65, p. 074031 (2002).

11. A. Bacchetta and M. Radici, Phys. Rev. D69, p. 074026 (2004).

12. A. Vossen et al., First Measurement of the Interference Fragmentation Function in $e^{+} e^{-}$at Belle, in Proceedings of XIII Advanced Research Workshop on High Energy Spin Physics, Dubna, Russia, Sept. 2009.

13. F. A. Ceccopieri, M. Radici and A. Bacchetta, Phys. Lett. B650, 81 (2007).

14. X. Artru and M. Mekhfi, Nucl. Phys. A532, 351 (1991).

15. P. J. Mulders and R. D. Tangerman, Nucl. Phys. B461, 197 (1996).

16. R. N. Cahn, Phys. Lett. B78, p. 269 (1978).

17. D. Boer and P. J. Mulders, Phys. Rev. D57, 5780 (1998).

18. H. Georgi and H. D. Politzer, Phys. Rev. Lett. 40, p. 3 (1978).

19. D. W. Sivers, Phys. Rev. D41, p. 83 (1990).

20. A. Kotzinian, Beyond Collins and Sivers: further measurements of the target transverse spin-dependent azimuthal asymmetries in semi-inclusive DIS from COMPASS, in Proceedings of DIS 2007, Munich, Germany, April 2007.

21. I. A. Savin, Azimuthal asymmetries in production of charged hadrons by high energy muons on polarized deuterium targets, in Proceedings of XIII Advanced Research Workshop on High Energy Spin Physics, Dubna, Russia, Sept. 2009.

22. H. Fischer, Review of Spin Physics in DIS, a personal selection, in Proceedings of DIS 2010, Florence, Italy, April 2010.

23. A. Airapetian et al., arXiv:1006.4221 (2010).

24. H. Wollny, Transversity Signal in two Hadron Pair Production in COMPASS, in Proceedings of DIS 2009, Madrid, Spain, April 2009.

25. R. Joosten, AIP Conf. Proc. 792, 957 (2005).

26. R. Joosten, AIP Conf. Proc. 915, 646 (2007).

27. A. Vossen, Measurement of Transverse Spin Effects at COMPASS, in Proceedings of 42 2nd Rencontres de Moriond on QCD and Hadronic Interactions, La Thuile, Italy, March 2007.

28. A. Ferrero, AIP Conf. Proc. 915, 436 (2007).

29. D. Kang, Longitudinal and transverse Lambda polarization at COMPASS, in Proceedings of DIS 2010, Florence, Italy, April 2010.

30. A. Airapetian et al., Phys. Rev. Lett. 103, p. 152002 (2009).

31. W. Kafer, Measurements of Unpolarized Azimuthal Asymmetries at COMPASS, in Proceedings of Transversity 2008, Ferrara, Italy, May 2008. 